\title{
A Modified Filtering Technique for Efficient Extreme Points and Application of MOLP in Agriculture Sector in Bangladesh
}

\author{
Md. Hasib Uddin Molla* and Md. Ainul Islam \\ Department of Mathematics, Dhaka University, Dhaka-1000, Bangladesh
}

(Received: 21 January 2016; Accepted: 18 May 2016)

\begin{abstract}
Multi-objective simplex algorithm computes all efficient extreme points of a multi-objective linear programming (MOLP) problem. For MOLP problem with large number of efficient extreme points, a filtering technique is necessary to present a small representative part of efficient extreme points. For this, we propose a filtering technique among efficient extreme points. In modern agriculture, we have to consider series of conflicting optimization criteria. For this reason, optimal cropping pattern, that is, allocation of land to various crops by making use of limited resources has become major challenges to get optimum return. To overcome this challenge, we illustrate the application of MOLP through a real life oriented problem of agricultural sector of Bangladesh. We also solve this problem by a self developed computer software which is based on multi-objective simplex algorithm and our proposed filtering technique.
\end{abstract}

Keywords: MOLP, Efficient Solutions, Multiobjective Simplex Method, Application of MOLP.

\section{Introduction}

A multi-objective linear programming (MOLP) problem is a generalized linear programming problem that involves multiple objective functions. The standard form of multiobjective linear programming problem is

$$
\min \{\boldsymbol{y}=C \boldsymbol{x}: A \boldsymbol{x}=\boldsymbol{b}, \boldsymbol{x} \geqq \mathbf{0}\}
$$

with a $(p \times n)$ objective (or criterion) matrix $C$ consisting of the rows $\boldsymbol{c}^{1}, \boldsymbol{c}^{2}, \ldots \ldots, \boldsymbol{c}^{p} ;(m \times n)$ constraint matrix $A$ consisting of the rows $\boldsymbol{a}^{1}, \boldsymbol{a}^{2}, \ldots \ldots, \boldsymbol{a}^{m}$; right hand side column vector $\boldsymbol{b} \in \mathbb{R}^{m}$ with $\boldsymbol{b} \geqq \mathbf{0}$ and decision variable vector $\boldsymbol{x} \in \mathbb{R}^{n} . \boldsymbol{y} \in \mathbb{R}^{p}$ is the objective function vector or criterion vector with $y_{i}=\boldsymbol{c}^{i} \boldsymbol{x}$ for $i=1,2, \ldots, p$ and we are minimizing the objective function vector.

In this section, we will state some basic definitions ${ }^{1,2,3}$ about MOLP.

Definition 1.1: The feasible set in the decision space is $\mathcal{X}=\left\{\boldsymbol{x} \in \mathbb{R}^{n}: A \boldsymbol{x}=\boldsymbol{b}, \boldsymbol{x} \geqq \mathbf{0}\right\}$. The feasible set in the objective space or criterion space is $\mathcal{Y}=C \mathcal{X}=\{\boldsymbol{y}=C \boldsymbol{x} \in$ $\left.\mathbb{R}^{p}: \boldsymbol{x} \in \mathcal{X}\right\}$.

Definition 1.2: Let $\hat{\boldsymbol{x}} \in \mathcal{X}$ be a feasible solution of the MOLP1 and let $\widehat{\boldsymbol{y}}=C \widehat{\boldsymbol{x}}$. Then

i) $\hat{x}$ is called weekly efficient solution if there is no $x \in \mathcal{X}$ such that $C \boldsymbol{x}<C \widehat{\boldsymbol{x}} ; \widehat{\boldsymbol{y}}$ is called weakly nondominated.

ii) $\hat{\boldsymbol{x}}$ is called efficient solution if there is no $\boldsymbol{x} \in \mathcal{X}$ such that $C \boldsymbol{x} \leq C \widehat{\boldsymbol{x}} ; \widehat{\boldsymbol{y}}$ is called non-dominated.

Definition 1.3: The set of all non-dominated criterion vector $\hat{\boldsymbol{y}} \in \mathcal{Y}$ is denoted by $\mathcal{Y}_{N}$ and called the non-dominated set. The set of all efficient solutions $\widehat{x} \in \mathcal{X}$ is denoted by $X_{E}$ and called the efficient set. If $\widehat{\boldsymbol{x}} \in \mathcal{X}_{E}$, then $\hat{\boldsymbol{y}}=C \widehat{\boldsymbol{x}} \in \mathcal{Y}_{N}$.

Definition 1.4: The weighted sum LP of MOLP1 is defined as follows:

$$
\min \left\{\boldsymbol{\lambda}^{T} C \boldsymbol{x}: A \boldsymbol{x}=\boldsymbol{b}, \boldsymbol{x} \geqq \mathbf{0}\right\}
$$

Definition 1.5: Let $\mathcal{B}$ be the set of indices of the basic variables of MOLP1. Then $\mathcal{N}:=\{1,2, \ldots, n\} \backslash \mathcal{B}$ is the set of nonbasic column indices. A variable $x_{i}$ and an index $i$ are called basic if $i \in \mathcal{B}$, nonbasic otherwise.

\section{Multiobjective Simplex Algorithm}

Various solution concepts for problem MOLP1 have been proposed. The concept of an efficient solution is commonly used. Many algorithms have been proposed to generate all of the efficient set or a representative portion, without any input from the decision maker and references therein.

Since the set of all dominated solutions is convex and the set of all non-dominated solutions is a subset of the convex hull of the non-dominated extreme points, P.L. Yu et $\mathrm{al}^{4}$ focus on how to generate the entire set of all non-dominated solutions through the set of all non-dominated extreme points. Issermann ${ }^{5}$ introduces an algorithm for the enumeration of the set of all efficient solutions for a linear multiple objective program. The procedure first finds whether an efficient solution exists for a given linear multiple objective program and then determines an initial efficient basic solution and finally the set of all efficient basic solutions. J.G. Ecker et $\mathrm{al}^{6}$ develop another method for finding all efficient extreme points for multiple objective linear programs. They gave a simple characterization of the efficiency of an edge incident to a non-degenerate or degenerate efficient vertex, which is the basis of their algorithm for enumeration of all efficient vertices. Using normal cones, N.T. Back Kim et $\mathrm{al}^{7}$ propose a method for generating efficient extreme points and efficient solution faces. They express optimality conditions in terms of negative normal cones.

Next, in this section we will discuss a generalized version of simplex method, named as multi-objective simplex algorithm to find all efficient extreme points of multiobjective linear programming problem. This is a non preference method which finds all efficient extreme points without any preference information from decision maker. The simplex algorithm for MOLP moves from one efficient basis to its adjacent efficient bases.

\footnotetext{
Author for correspondence. e-mail: hasib.math@du.ac.bd
} 
Before starting the discussion of the multiobjective simplex algorithm we will state some related well known theorems ${ }^{1,2,3,4}$, related to MOLP.

Theorem 2.1: The feasible sets $\mathcal{X}$ in decision space and objective space $\mathcal{Y}$ of the MOLP1 are convex and closed.

Theorem 2.2: Let $\widehat{\boldsymbol{x}} \in \mathcal{X}$ be an optimal solution of weighted sum LP given by LP $(\boldsymbol{\lambda})$. Then

1. if $\boldsymbol{\lambda} \geq \mathbf{0}$ then $\hat{\boldsymbol{x}}$ is weakly efficient.

2. if $\boldsymbol{\lambda}>\mathbf{0}$ then $\widehat{\boldsymbol{x}}$ is efficient.

Lemma 2.3: A feasible solution $x^{0} \in \mathcal{X}$ of MOLP1 is efficient if and only if the linear program

$\max \left\{\boldsymbol{e}^{T} \boldsymbol{z}: A \boldsymbol{x}=\boldsymbol{b}, C \boldsymbol{x}+I \boldsymbol{z}=C \boldsymbol{x}^{0}, \boldsymbol{x}, \boldsymbol{z} \geqq \mathbf{0}\right\}$

where $\boldsymbol{e}^{T}=(1,1, \ldots, 1) \in \mathbb{R}^{p}$ and $I$ is the $p \times p$ identity matrix, has an optimal solution $(\widehat{\boldsymbol{x}}, \hat{\mathbf{z}})$ with $\widehat{\mathbf{z}}=\mathbf{0}$.

Lemma 2.4: A feasible solution $\boldsymbol{x}^{0} \in \mathcal{X}$ of MOLP1 is an efficient solution if and only if the linear program

$$
\min \left\{\boldsymbol{u}^{T} \boldsymbol{b}+\boldsymbol{w}^{T} C \boldsymbol{x}^{0}: \boldsymbol{u}^{T} A+\boldsymbol{w}^{T} C \geqq \mathbf{0}, \boldsymbol{w} \geqq \boldsymbol{e}, \boldsymbol{u} \in \mathbb{R}^{m}\right\}
$$

has an optimal solution $(\widehat{\boldsymbol{u}}, \widehat{\boldsymbol{w}})$ with $\widehat{\boldsymbol{u}}^{T} \boldsymbol{b}+\widehat{\boldsymbol{w}}^{T} C \boldsymbol{x}^{0}=0$.

Theorem 2.5: A feasible solution $\boldsymbol{x}^{0} \in \mathcal{X}$ of MOLP1 is an efficient solution if and only if there exists a $\lambda \in \mathbb{R}^{p}$ such that $\boldsymbol{x}^{0}$ is an optimal solution of the LP $(\boldsymbol{\lambda})$ which is the weighted sum LP of the MOLP1 with weighting vector $\lambda>\mathbf{0}$.

Theorem 2.6: If $X$ has an efficient point, then at least one extreme point of $\mathcal{X}$ is efficient.

Theorem 2.7: Let $\mathcal{B}$ be an efficient basis and $\left(\boldsymbol{x}_{\mathcal{B}}, \mathbf{0}\right)$ be the corresponding basic feasible solution. Then $\left(\boldsymbol{x}_{\mathcal{B}}, \mathbf{0}\right)$ is an efficient extreme point of $\chi_{E}$.

Theorem 2.8: Let $\boldsymbol{x} \in \mathcal{X}_{E}$ be an extreme point. Then there exists an efficient basis $\mathcal{B}$ such that $\boldsymbol{x}=\left(\boldsymbol{x}_{\mathcal{B}}, \mathbf{0}\right)$.

Theorem 2.9: Let $\mathcal{B}$ be an efficient basis and $x_{j}$ be a nonbasic variable. Let $R$ be nonbasic part of the reduced cost matrix of MOLP1 with respect to the basis $\mathcal{B}$. Variable $x_{j}$ is an efficient nonbasic variable if and only if the LP

$$
\begin{gathered}
\max \left\{\boldsymbol{e}^{T} \boldsymbol{v}: R \boldsymbol{z}-\boldsymbol{r}^{j} \delta+I \boldsymbol{v}=\mathbf{0}, \mathbf{0} \leqq \boldsymbol{z} \in \mathbb{R}^{n-m}, 0 \leqq \delta\right. \\
\left.\in \mathbb{R}, \mathbf{0} \leqq \boldsymbol{v} \in \mathbb{R}^{p}\right\}
\end{gathered}
$$

has an optimal value of 0 .

Theorem 2.10: All efficient bases are connected.

Theorem 2.11: $x_{E}$ is connected and, therefore $y_{N}$ is connected

For the problem MOLP1 one and only one of the following cases can occur:

1. MOLP1 is infeasible, that is, $\chi=\emptyset$.

2. MOLP1 is feasible $(\mathcal{X} \neq \varnothing)$ but has no efficient solutions $\left(\chi_{E}=\emptyset\right)$, or

3. MOLP1 is feasible and has efficient solutions, that is, $x_{E} \neq \varnothing$.
Multi-objective simplex algorithm deals with these situations. Multi-objective simplex algorithm for computing all efficient extreme points possesses the following three phases:

Phase I: Determine an initial basic feasible solution or stop with the conclusion that $\mathcal{X}=\varnothing$.

Phase II: Determine an initial efficient basis or stop with the conclusion that $\chi_{E}=\emptyset$.

Phase III: Pivot among efficient bases to determine all efficient bases.

Phase I in here is the ordinary Phase I from Simplex method of Linear Programming $8,9,10,11$.

For finding an initial efficient basis in Phase II, several approaches are available. We will discuss one of them that use weighted sum approach.

The solution of a weighted sum LP given by LP $(\boldsymbol{\lambda})$ with $\boldsymbol{\lambda}>\mathbf{0}$ will yield an efficient basis for MOLP1, provided LP $(\lambda)$ is bounded. If we do not know that in advance, it is necessary to come up with a procedure that either concludes that $\chi_{E}=\varnothing$ or returns an appropriate $\lambda$ for which LP $(\lambda)$ has an optimal solution. Assuming that $\mathcal{X} \neq \varnothing$, then Phase I returns a basic feasible solution $\boldsymbol{x}^{0} \in \mathcal{X}$, which may or may not be efficient. We proceed in two steps ${ }^{1,2,4,5}$ :

First, the auxiliary LP 2.4 is solved to check whether $\chi_{E}=$ $\emptyset$. $\chi_{E} \neq \emptyset$ if and only if LP 2.4 has an optimal solution. Next we consider the following lemma.

Lemma 2.12: If $X_{E} \neq \varnothing$ the optimal solution of LP 2.4 returns an appropriate weighting vector $\widehat{\boldsymbol{w}}$ for which LP $(\boldsymbol{\lambda})$ will have an optimal solution.

So it follows that LP 2.4 and LP $(\widehat{\boldsymbol{w}})$ are the necessary tools in Phase II. If LP 2.4 is infeasible, $\chi_{E}=\emptyset$. Otherwise an optimal solution of LP 2.4 yields an appropriate weighting vector $\widehat{\boldsymbol{w}}$ for which LP $(\widehat{\boldsymbol{w}})$ has an optimal basic feasible solution, which is an initial efficient basic feasible solution of the MOLP1.

For determining all efficient bases of MOLP1, the strategy is to pursue each nonbasic variable in each efficient basis to check if pivoting with this nonbasic variable as entering into the basis will leads to a new efficient basic feasible solution. Once we have an initial efficient basis $\mathcal{B}$, we start with a nonbasic variable $x_{j} ; j \in \mathcal{N}$ at $\mathcal{B}$ as entering variable. Then by usual pivoting procedure of simplex method we will find a new basis $\widehat{\mathcal{B}}$ adjacent to $\mathcal{B}$. Now we need to check whether this new basis $\widehat{\mathcal{B}}$ is efficient. Now to check whether this new basis $\widehat{\mathcal{B}}$ is efficient for MOLP1 we will use EvansSteuer Test ${ }^{2}$. There are also other tests available, which are introduced by Bach Kim et $\mathrm{al}^{12}$ and P.L. Yu et $\mathrm{al}^{4}$.

Evans-Steuer test: In this test we will check if a basis $\widehat{\mathcal{B}}$ which is obtained from an efficient basis $\mathcal{B}$ by a single pivot is efficient or not. Suppose $\widehat{\mathcal{B}}$ is obtained from $\mathcal{B}$ by selecting $x_{j}$ as entering variable, where $x_{j}$ is nonbasic at $\mathcal{B}$. Since if $x_{j}$ is an efficient nonbasic variable at $\mathcal{B}$, then any feasible pivot from $\mathcal{B}$ with $x_{j}$ entering into the basis will 
leads to an adjacent efficient basis $\widehat{\mathcal{B}}$. So we need only to check that if $x_{j}$ is an efficient nonbasic variable at $\mathcal{B}$. We can check this using Theorem 2.9 which is known as EvansSteuer test. If $x_{j}$ is an efficient nonbasic variable at $\mathcal{B}$, then $\widehat{\mathcal{B}}$ which is obtained from $\mathcal{B}$ by selecting $x_{j}$ as entering variable is an efficient basis of MOLP1.

\section{Multi-objective Simplex Algorithm:}

Step 1: Determine an initial basic feasible solution of MOLP1 by Phase I of the two phase method for linear programming problem. If an initial basic feasible solution cannot be found then MOLP1 is infeasible and algorithm stops.

Step 2: Solve LP 2.4. If this problem is infeasible, then $\chi_{E}=\varnothing$ and algorithm stops. Otherwise let $(\widehat{\boldsymbol{u}}, \widehat{\boldsymbol{w}})$ be an optimal solution. Now move to the next step.

Step 3: Find an optimal basis $\mathcal{B}_{1}$ of the $\operatorname{LP}(\boldsymbol{\lambda})$ with weighting vector $\lambda=\widehat{\boldsymbol{w}}$, that is, LP $(\widehat{\boldsymbol{w}})$. This basis $\mathcal{B}_{1}$ is our initial efficient basis of MOLP1. Let $\mathcal{N}_{1}:=\{1,2, \ldots, n\} \backslash \mathcal{B}_{1}$ be the set of nonbasic column indices at $\mathcal{B}_{1}$. Set $\mathcal{L}:=\left\{\mathcal{B}_{1}\right\}$, bcount $=1$ and $i=1$.

Step 4: For all $j \in \mathcal{N}_{i}$ where $\mathcal{N}_{i}:=\{1,2, \ldots, n\} \backslash \mathcal{B}_{i}$ is the set of nonbasic column indices for basis $\mathcal{B}_{i}$, repeat step 4(a) to 4(b).

a) With $x_{j}$ as entering variable at $\mathcal{B}_{i}$, by usual pivoting procedure of simplex method find a basis $\widehat{\mathcal{B}}$ adjacent to $\mathcal{B}$. Now we need to check whether this new basis $\widehat{\mathcal{B}}$ is efficient or not. If $\widehat{\mathcal{B}}$ is efficient then move to the step 4(b) otherwise start step 4(a) with next $j \in \mathcal{N}_{i}$.

b) If $\mathcal{L} \cap \widehat{\mathcal{B}}=\emptyset$, then bcount $=$ bcount $+1, \mathcal{B}_{\text {bcount }}:=$ $\widehat{\mathcal{B}}$ and $\mathcal{L}:=\mathcal{L} \cup \mathcal{B}_{\text {bcount }}$.

Step 5: Set $i=i+1$. If $i \leqq b$ count then go to step 4 otherwise go to next step.

Step 6: All the efficient bases of MOLP1 is obtained. Output bcount the total number of efficient extreme points and $\mathcal{L}$, set of all efficient extreme points of MOLP1

\section{Filtering Among the Efficient Extreme Points}

Filtering refers to the process of selecting subset of points from a larger finite set of points. For MOLP problem with large number of efficient points, it becomes very difficult for decision maker to examine all the efficient points physically. There are two filtering techniques discussed by Steuer ${ }^{2}$. J.G. Ecker et al $^{13}$ propose another method for selecting certain subsets from the set $\mathcal{Y}_{N}$ of non-dominated points for multi-objective linear programming problems. One such subset is the set of a point $\boldsymbol{y} \in \mathcal{Y}_{N}$ for which the maximum deviation of the objective function values from some ideal vector $\boldsymbol{m}$ is as small as possible. Proposed method does not require that the set $\mathcal{Y}_{N}$ be calculated explicitly and subsets can be obtained as the set of nondominated points.

In this section, we propose a new filtering technique that will represent atmost $(p+1)$ efficient extreme points for an MOLP with $p$ objectives.
The idea behind this new filtering technique is that, for efficient point when one objective function attains its best value, then other objectives attain their comparatively worst values. So we are looking for compromised solution, where none of the objectives attain their comparatively worst values.

Suppose Nex is the set of non-dominated points corresponding to the efficient extreme points. Then for any $\boldsymbol{v} \in_{\text {Nex }}$ we have $\boldsymbol{v}=\left(v_{1}, v_{2}, \ldots, v_{p}\right)$. Now for each $i=$ $1,2, \ldots, p$ we calculate

$$
Q_{i}=\frac{\max _{v \in \text { Nex }}\left\{v_{i}\right\}+\min _{v \in \text { Nex }}\left\{v_{i}\right\}}{2}
$$

Then for each $i=1,2, \ldots, p$ we select an efficient extreme point whose corresponding criterion vector has its $i$ th component smallest distance from $Q_{i}$. That is, for each $i=1,2, \ldots, p$ we select an efficient extreme point whose corresponding criterion vector $\boldsymbol{v}$ has the property:

$$
\min _{v \in \in_{N e x}}\left|v_{i}-Q_{i}\right|
$$

In this way we will find $p$ efficient extreme points.

For another efficient extreme point, first set $\boldsymbol{v}^{*}=$ $\left(v_{1}^{*}, v_{2}^{*}, \ldots, v_{p}^{*}\right)$, where $v_{i}^{*}=\min _{v \in \text { Nex }}\left\{v_{i}\right\}$. Then we will find a criterion vector $\boldsymbol{v} \in_{\text {Nex }}$ so that $\boldsymbol{v}$ has the smallest distance from $v^{*}$. For measuring distance we will consider weighted $L_{2}$-metric. In accordance with a $L_{2}$-metric, the distance between two criterion vector $\boldsymbol{v}^{1}$ and $\boldsymbol{v}^{2}$ is given by

$$
\left\|\boldsymbol{v}^{1}-\boldsymbol{v}^{2}\right\|^{\boldsymbol{\pi}}=\sqrt{\left[\sum_{i=1}^{p}\left(\pi_{i}\left|v_{i}^{1}-v_{i}^{2}\right|\right)^{2}\right]}
$$

Where $\pi_{i}$ is the range equalization weight associated with the $i$ th component of the criterion vector. The purpose of the $\pi_{i}$ weights is to equalize the ranges of the components of the criterion vectors, so that the filtering process will not be biased in favor of the components with the largest range. The range equalization weight $\pi_{i}$ is calculated by

$$
\pi_{i}=\frac{1}{R_{i}}\left[\sum_{j=1}^{p} \frac{1}{R_{j}}\right]^{-1}
$$

where, $R_{i}=\max _{v \in_{N e x}}\left\{v_{i}\right\}-\min _{v \in_{N e x}}\left\{v_{i}\right\}$

In this way we filter among efficient extreme points to find a small but useful representative part of the efficient extreme points so that decision maker can compare and choose one of them easily and effectively.

In case of large scale multi-objective linear programming problem we need a computer technique for computing efficient extreme points and performing filter. So based on multiobjective simplex algorithm and above proposed filtering technique we have built a computer software. 


\section{Application of MOLP in Agricultural Sector of Bangladesh}

Several researchers including Asis ${ }^{14}$, Kumari ${ }^{15}$ and Herath ${ }^{16}$ studied the application of multi-objective linear programming in agricultural production planning under uncertain environments. In our agricultural model we consider specific value for the requirements, capacity power of market and resources.

Problem: Suppose we have 11 crops to be cultivated for winter season in Magura district of Bangladesh. Requirements of water, workers, fertilizers and estimated revenue of the crops per acres are given. Also the available resources and capacity power of markets are given.

Table 1. Requirement and estimated revenue

\begin{tabular}{|c|c|c|c|c|c|c|c|}
\hline & Name & $\begin{array}{l}\text { Wat } \\
\text { er } \\
\text { (Ho } \\
\text { ur) }\end{array}$ & $\begin{array}{l}\text { Wor } \\
\text { ker } \\
\text { (Day } \\
\text { ) }\end{array}$ & $\begin{array}{l}\text { Urea } \\
(\mathrm{kg})\end{array}$ & $\begin{array}{l}\text { T.S } \\
. P . \\
(\mathrm{kg} \\
)\end{array}$ & $\begin{array}{l}\text { Pot } \\
\text { as } \\
(\mathrm{kg})\end{array}$ & $\begin{array}{c}\text { Reven } \\
\text { ue } \\
\text { (Thou } \\
\text { sand } \\
\text { Tk) }\end{array}$ \\
\hline 1 & Cabbage & 100 & 54 & 133 & 107 & 24 & 100 \\
\hline 2 & $\begin{array}{c}\text { Cauliflo } \\
\text { wer }\end{array}$ & 100 & 54 & 113 & 142 & 48 & 90 \\
\hline 3 & Carrot & 36 & 60 & 100 & 93 & 40 & 15 \\
\hline 4 & Potato & 30 & 54 & 139 & 71 & 55 & 60 \\
\hline 5 & Mustard & 18 & 66 & 77 & 57 & 24 & 25.5 \\
\hline 6 & Brinjal & 80 & 50 & 123 & 86 & 36 & 140 \\
\hline 7 & Rice & 120 & 48 & 138 & 36 & 30 & 37.5 \\
\hline 8 & Wheat & 18 & 42 & 123 & 71 & 36 & 29.75 \\
\hline 9 & $\begin{array}{l}\text { Pulse1 } \\
\text { (Lentil) }\end{array}$ & 12 & 36 & 19 & 71 & 15 & 31.5 \\
\hline $\begin{array}{l}1 \\
0\end{array}$ & $\begin{array}{c}\text { Pulse } 2 \\
\text { (Chickpe } \\
\text { a) }\end{array}$ & 12 & 36 & 15 & 45 & 15 & 30 \\
\hline $\begin{array}{l}1 \\
1\end{array}$ & $\begin{array}{c}\text { Pulse } 3 \\
\text { (Mungbe } \\
\text { an) }\end{array}$ & 18 & 36 & 18 & 71 & 14 & 36 \\
\hline
\end{tabular}

Available resources are

1. Total available area for winter crops $\leqq 80,000$ Acres

2. The available irrigation water $\leqq 48,00,000$ Hours

3. Available Urea $\leqq 80,00,000 \mathrm{~kg}$

4. Available T.S.P. $\leqq 60,00,000 \mathrm{~kg}$

5. Available Potas $\leqq 28,00,000 \mathrm{~kg}$

The capacity powers of markets are
1. Cabbage + Cauliflower + Carrot + Bringal $\geqq 15000$ Acres

2. Pulse $1+$ Pulse $2+$ Pulse $3 \geqq 18000$ Acres

3. Potato $\geqq 8000$ Acres

4. Mustard $\geqq 5000$ Acres

5. Rice $\geqq 12000$ Acres

6. Wheat $\geqq 5000$ Acres

Now we want to find out a planning that how much land can be used for each crop so that it maximize revenue, minimizes the quantity of irrigation water, Urea, TSP, Potas and number of workers.

MOLP Model Formulation: To build a MOLP model from the above problem we need to define decision variables first, then formulate all possible objective functions and constraints from imposed conditions.

We define decision variables as follows:

$x_{i}=$ Number of acres to be used for crop $i ; i=1,2, \ldots, 11$.

Since we want to maximize revenue, using revenue from given table, one objective function is

$$
\begin{aligned}
\max 100 x_{1}+ & 90 x_{2}+15 x_{3}+60 x_{4}+25.5 x_{5}+140 x_{6} \\
& +37.5 x_{7}+29.75 x_{8}+31.5 x_{9}+30 x_{10} \\
& +36 x_{11}
\end{aligned}
$$

which is equivalent to

$$
\begin{aligned}
\min -\left(100 x_{1}+\right. & 90 x_{2}+15 x_{3}+60 x_{4}+25.5 x_{5}+140 x_{6} \\
& +37.5 x_{7}+29.75 x_{8}+31.5 x_{9}+30 x_{10} \\
& \left.+36 x_{11}\right)
\end{aligned}
$$

Since we also want to minimizes the quantity of irrigation water needed, number of workers and quantity of urea, TSP and Potas being used, in similar way we can formulate the corresponding objective functions respectively.

According to the available resources and capacity power of markets we can formulate the relative constraints also.

Combining objective functions, related constraints and then introducing necessary slack and surplus variables we get the following standard form of MOLP model associated to our problem:

$$
\begin{aligned}
& \min \quad \boldsymbol{y}=C \boldsymbol{x} \\
& \text { Subject to } A \boldsymbol{x}=\boldsymbol{b} \\
& \boldsymbol{x} \geqq \mathbf{0}
\end{aligned}
$$

where $\boldsymbol{x}=\left(x_{1}, x_{2}, \ldots, x_{22}\right)$ and $\boldsymbol{b}, C$ and $A$ are given by

$$
\boldsymbol{b}=\left(\begin{array}{lllllllllll}
4800000 & 8000000 & 6000000 & 2800000 & 80000 & 15000 & 18000 & 8000 & 5000 & 12000 & 5000
\end{array}\right)^{\mathrm{T}}
$$




$$
\begin{aligned}
C= & \left(\begin{array}{ccccccccccccccccccccccc}
-100 & -90 & -15 & -60 & -25.5 & -140 & -37.5 & -29.75 & -31.5 & -30 & -36 & 0 & 0 & 0 & 0 & 0 & 0 & 0 & 0 & 0 & 0 & 0 \\
100 & 100 & 36 & 30 & 18 & 80 & 120 & 18 & 12 & 12 & 18 & 0 & 0 & 0 & 0 & 0 & 0 & 0 & 0 & 0 & 0 & 0 \\
54 & 54 & 60 & 54 & 66 & 50 & 48 & 42 & 36 & 36 & 36 & 0 & 0 & 0 & 0 & 0 & 0 & 0 & 0 & 0 & 0 & 0 \\
133 & 113 & 100 & 139 & 77 & 123 & 138 & 123 & 19 & 15 & 18 & 0 & 0 & 0 & 0 & 0 & 0 & 0 & 0 & 0 & 0 & 0 \\
107 & 142 & 93 & 71 & 57 & 86 & 36 & 71 & 71 & 45 & 71 & 0 & 0 & 0 & 0 & 0 & 0 & 0 & 0 & 0 & 0 & 0 \\
24 & 48 & 40 & 55 & 24 & 36 & 30 & 36 & 15 & 15 & 14 & 0 & 0 & 0 & 0 & 0 & 0 & 0 & 0 & 0 & 0 & 0
\end{array}\right) \\
\mathrm{A} & =\left(\begin{array}{cccccccccccccccccccccccc}
100 & 100 & 36 & 30 & 18 & 80 & 120 & 18 & 12 & 12 & 18 & 1 & 0 & 0 & 0 & 0 & 0 & 0 & 0 & 0 & 0 & 0 \\
133 & 113 & 100 & 139 & 77 & 123 & 138 & 123 & 19 & 15 & 18 & 0 & 1 & 0 & 0 & 0 & 0 & 0 & 0 & 0 & 0 & 0 \\
107 & 142 & 93 & 71 & 57 & 86 & 36 & 71 & 71 & 45 & 71 & 0 & 0 & 1 & 0 & 0 & 0 & 0 & 0 & 0 & 0 & 0 \\
24 & 48 & 40 & 55 & 24 & 36 & 30 & 36 & 15 & 15 & 14 & 0 & 0 & 0 & 1 & 0 & 0 & 0 & 0 & 0 & 0 & 0 \\
1 & 1 & 1 & 1 & 1 & 1 & 1 & 1 & 1 & 1 & 1 & 0 & 0 & 0 & 0 & 1 & 0 & 0 & 0 & 0 & 0 & 0 \\
1 & 1 & 1 & 0 & 0 & 1 & 0 & 0 & 0 & 0 & 0 & 0 & 0 & 0 & 0 & 0 & 1 & 0 & 0 & 0 & 0 & 0 \\
0 & 0 & 0 & 0 & 0 & 0 & 0 & 0 & 1 & 1 & 1 & 0 & 0 & 0 & 0 & 0 & 0 & -1 & 0 & 0 & 0 & 0 \\
0 & 0 & 0 & 1 & 0 & 0 & 0 & 0 & 0 & 0 & 0 & 0 & 0 & 0 & 0 & 0 & 0 & 0 & -1 & 0 & 0 & 0 \\
0 & 0 & 0 & 0 & 1 & 0 & 0 & 0 & 0 & 0 & 0 & 0 & 0 & 0 & 0 & 0 & 0 & 0 & 0 & -1 & 0 & 0 \\
0 & 0 & 0 & 0 & 0 & 0 & 1 & 0 & 0 & 0 & 0 & 0 & 0 & 0 & 0 & 0 & 0 & 0 & 0 & 0 & -1 & 0 \\
0 & 0 & 0 & 0 & 0 & 0 & 0 & 1 & 0 & 0 & 0 & 0 & 0 & 0 & 0 & 0 & 0 & 0 & 0 & 0 & 0 & -1
\end{array}\right)
\end{aligned}
$$

Here in standard form, total number of variables are 22 , number of constraints are 11 and number of objectives are 6. In the original problem number of decision variables were 11.

Filtered file contains the following information:

Objective 1 has maximum value -1971250.000 at $\boldsymbol{x}(6)$ and minimum value -6306516.667 at $\boldsymbol{x}(24)$

Objective 2 has maximum value $\quad 4800000.000$ at $x(11)$ and minimum value 2616000.000 at $x(2)$

Objective 3 has maximum value $\quad 3828290.323$ at $\boldsymbol{x}(23)$ and minimum value 2946000.000 at $\boldsymbol{x}(3)$

Objective 4 has maximum value 8000000.000 at $x(9)$ and minimum value 5538000.000 at $\boldsymbol{x}(6)$

Objective 5 has maximum value 5727450.602 at $\boldsymbol{x}(20)$ and minimum value 3740000.000 at $\boldsymbol{x}(7)$

Objective 6 has maximum value 2765645.161 at $x(23)$ and minimum value 1712000.000 at $\boldsymbol{x}(5)$

Filtered efficient extreme points are:

$\begin{array}{clllllllllll}\boldsymbol{x}(8)= & 0.0 & 0.0 & 0.0 & 8000 & 5000 & 15000 & 12000 & 5000 & 0.0 & 0.0 & 18000 \\ \boldsymbol{x}(16)= & 0.0 & 0.0 & 0.0 & 8000 & 5000 & 15000 & 12000 & 5000 & 0.0 & 0.0 & 35000 \\ \boldsymbol{x}(10)= & 0.0 & 0.0 & 0.0 & 8000 & 5000 & 15000 & 12000 & 5000 & 35000 & 0.0 & 0.0 \\ \boldsymbol{x}(12)= & 0.0 & 15000 & 0.0 & 8000 & 5000 & 0.0 & 12000 & 5000 & 0.0 & 18000 & 0.0 \\ \boldsymbol{x}(7)= & 0.0 & 0.0 & 0.0 & 8000 & 5000 & 15000 & 12000 & 5000 & 0.0 & 18000 & 0.0\end{array}$

Corresponding non-dominated points are:

$\begin{array}{cllllll}\boldsymbol{y}(8)= & -3954250 & 3384000 & 2946000 & 5937000 & 4208000 & 1892000 \\ \boldsymbol{y}(16)= & -4566250 & 3690000 & 3558000 & 6243000 & 5415000 & 2130000 \\ \boldsymbol{y}(10)= & -4408750 & 3480000 & 3558000 & 6278000 & 5415000 & 2165000 \\ \boldsymbol{y}(12)= & -3096250 & 3576000 & 3006000 & 5733000 & 4580000 & 2090000 \\ \boldsymbol{y}(7)= & -3846250 & 3276000 & 2946000 & 5883000 & 3740000 & 1910000\end{array}$

Now it will become easy for decision maker to compare and select a planning of land for implementation after observing the above informations. Observing the above non-dominated points we can say that efficient extreme point $\boldsymbol{x}(7)$ or $\boldsymbol{x}(8)$ are comparatively reasonable for practical use because none of the objectives are close to their worst value. In $x(16)$ or $\boldsymbol{x}(10)$ the value of the first objective function is better than of $\boldsymbol{x}(7)$ or $\boldsymbol{x}(8)$, but value of the other objective functions are worst in $\boldsymbol{x}(16)$ or $\boldsymbol{x}(10)$ than of $\boldsymbol{x}(7)$ or $\boldsymbol{x}(8)$. 


\section{Conclusion}

In this paper we consider real life oriented problem of agricultural sector of Bangladesh to illustrate the application of MOLP. We see that the multi-objective simplex algorithm along with our filtering technique can be useful for the comparative study of real life oriented problems involves with multiple objectives. Multi-objective simplex algorithm computes all efficient extreme points and filtering technique enables the decision maker to choose one of the point from a small representative part of efficient extreme points. In this process our software helps us to reduce the computing effort. Agriculture model in this paper is designed for a particular season. Year wise plan for allocation of land to various crops is possible to optimize the objectives. Then one has to consider time constraints also, so that accommodation for maximum number of crops throughout a year is possible. Further research can be done taking the uncertainties into account, considering both $\leq$ and $\geq$ type constraints for requirements and capacity power of market.

\section{References}

1. Ehrgott, M., 2005. Multicriteria Optimization. $2^{\text {nd }} E d$, Springer.

2. Steuer, Ralph E., 1985. Multicriteria Optimization - Theory, Computation and Application. Wiley.

3. Zeleny, M., 1982. Multiple Criteria Decision Making. McGraw-Hill.

4. Yu, P. L. and Zeleny, M., 1975. The set of all nondominated solutions in linear cases and a multicriteria simplex method. Journal of Mathematical Analysis and Applications. 49, 430-468.

5. Isermann, H., 1977. The enumeration of the set of all efficient solutions for a linear multiple objective program. Operational Research Quarterly. 28(3), 711-725.

6. Ecker, J.G. and Kouda, I.A., 1978. Finding all Efficient Extreme Points for Multiple Objective Linear Programs. Mathematical Programming, 14, 249-261.
7. Back Kim, N.T. and Luc, D.T., 2000. Normal Cones to a Polyhedral Convex Set and Generating Efficient Faces in Linear Multi-objective Programming. ACTA Mathematica Vietnamica, 25(1), 101-124.

8. Hillier, Frederick S. and Lieberman, Gerald J., 2001. Introduction to Operations Research. $7^{\text {th }}$ Ed., McGraw-Hill.

9. Kambo, NS, 1991. Mathematical Programming Techniques. Affiliated East West Press, Newdelhi.

10. Raavindran, A., Phillips, Don T. and Solberg, James J., 1987. Operation Research: Principle and Practise. $2^{\text {nd }}$ Ed., Wiley.

11. Taha, Hamdy A., 2007. Operations Research: An introduction. $8^{\text {th }}$ Ed., Pearson Princeton Hall.

12. Bach Kim, N. T., Thien, N. T. and Thuy, L. Q., 2008. Generating all efficient extreme solutions in multiple objective linear programming problem and its application. East-West J. of Mathematics, 10(1), 1-14.

13. Ecker, J. G. and Shoemaker, Nancy E, 1981. Selecting subsets from the set of nondominated vectors in multiple objective linear programming. Siam J. Control and Optimization. 19(4), 505-515.

14. Asis, Sayed Abdel, 2007. Parametric and Multiobjective Optimisation Applied in Agriculture: The Study of Cropping Pattern in the Ameriya Region in Winter Crops. Revista Investigacion Operacional, 28(1),17-24.

15. Kumari, P. Lavanya, Reddy, G. Krisna and Krisna, T.Giridhara, Optimum Allocation of Agriculture Land to the Vegetable Crops under Uncertain Profits using Fuzzy Multiobjective Linear Programming. IOSR Journal of Agriculture and Veterinary Science, 7(12), 19-28.

16. Herath, H.M.I.U and D. M.,, Samarathunga 2015. MultiObjective Fuzzy Linear Programming in Agricultural Production Planning. International Journal of Scientific \& Technology Research, October 4(10), 242-250. 
